Pure and Applied Mathematics Quarterly

Volume 5, Number 4

(Special Issue: In honor of

John Tate, Part 1 of 2)

$1429-1433,2009$

\title{
The Mathematical Circle Around Tate In The Late Fifties and Early Sixties
}

\author{
Stephen S. Shatz \\ Abstract: A discussion from the personal and historical point of view of \\ the graduate mathematics students and the mathematical activity around \\ John Tate at Harvard in the period: 1958-1962.
}

Keywords: History, graduate mathematics, algebraic geometry, number theory, John T. Tate, Jr.

After three years as an instructor at Princeton (1950-1953) and one year as an assistant professor at Columbia ('53-'54), John Tate came to Harvard in the autumn of 1954. He was already known among mathematicians for his share of the famous Artin-Tate Seminar, for his thesis (yet unpublished) and for his complete clarification of the cohomological structure of class field theory (for which he would be awarded the AMS Cole Prize in Number Theory-1956). I met him that autumn, but I was still only a sophomore in college and still a physics student then. Nonetheless, there was about him already an aura that attracted many people including myself though I would have been hard pressed then to say why. My switch to mathematics came from some inner compulsion as well as being befriended by Richard Brauer and Lars Ahlfors. Some people have a great deal of luck!

Bernard Dwork also came to Harvard that autumn and, while he was officially a student of E. Artin and thus Tate's mathematical brother, he really considered

Received November 30, 2006. 
himself a Tate student (the first in what would be a long line). In any case, Tate and Dwork were already very close and had many animated discussions in a little seminar room where we sometimes had tea. I was extremely fortunate to make friends with Dwork and learn a great deal from him while still an undergraduate; as I said, some people have a great deal of luck. At the time, Dwork must have been formulating his deep studies of p-adic analysis which led to his proof of the rationality of the Weil Zeta function of a variety over a finite field. Many times I caught suggestive words as I passed by this little room: root number, functional equation, Frobenius, Euler product, etc. It is impossible to indicate the excitement that radiated from the two of them in this little room and I was not alone in receiving it.

One must remember that this was also the time that Jean-Pierre Serre began his continued visits to Harvard, sometimes for a year, more normally for one of the two semesters. Serre and Tate had become fast friends and the atmosphere was quite charged when they were both around-though it was hard to get to see Tate then as he was very busy with Serre.

At Harvard, Tate's first student was Edward Assmus. He graduated in 1958 and so I do not know about his participation in what may have been a small circle around Tate in the middle fifties. He did not participate in the growing circle of slightly younger students that was forming around Tate about the middle of 1958. The younger students who formed the group around Tate were some of his own and some working with other advisors-principally Zariski, though at least one of Mackey's students was involved. Here are the names of those students, listed in order of their degree dates and with their advisors also listed: Michael Artin (1960-Zariski), Leonard Evens (1960-Tate), Calvin Moore (1960-Mackey), James Cohn (1961-Tate), Andrew Ogg (1961-Tate), Stephen Shatz (1962-Tate), Jonathan Lubin (1963-Tate), Judith Obermayer (1963-Tate), Stephen Lichtenbaum (1964-Tate), J. Michael Schlessinger (1964-Tate), John McCabe (1967Tate). Occasionally, we had the participation of Heisuke Hironaka (1960-Zariski) and David Mumford (1961-Zariski) as well as Warren Wong (1959-Brauer) and Morton Harris (1960-Brauer). Students from MIT were also occasionally involved, but the principal participants were from Harvard. As one can imagine, this was a lively group; there were even "secret seminars" in which one could let 
one's hair down and confess ignorance or expose the struggling we all were going through to master hard material. The seminars were secret only in that we took care not to invite faculty!

The year 1957-58 found Tate in Paris where he interacted with Serre and doubtless with Grothendieck. I recall a letter from Tate to Evens from approximately this time in which the Atiyah-Hirzebruch Spectral Sequence was discussed. Evens, then becoming an expert on the cohomology ring of a finite group, was to prove that this ring, with cup product as its multiplication, is noetherian. However, the point of my recollection is that already the nascent group around Tate was functioning even though the leader was away in Paris.

The year 1959-60 was a banner year both for Harvard's Mathematics Department and for the Tate circle. During that time Akizuki, Grothendieck and Nagata were in residence the entire year. Actually, due to some visa problems, Grothendieck arrived about one and a half months late. And so, Tate was the lecturer in the graduate course that Grothendieck had announced: Theory of Sheaves. What an audience was in attendance! Of course, there was the usual complement of graduate students, but the first row was filled with dignitaries: Akizuki, Nagata, Zariski, Lang (when he was in town), Tate (when he had stopped filling in for Grothendieck). We were going to learn scheme theory from Grothendieck in a seminar and, for that, we really had to know sheaves. In addition, there was another course with a powerhouse audience: Bott had arrived from Michigan and gave an introductory graduate course on Algebraic Topology, but everybodysimply everybody-had to hear Bott on Algebraic Topology. It was during this year, too, that Tate and Bott formed their fast friendship.

Actually, the year '59-'60 began in the summer when the Tate circle held its first summer seminar. These seminars ran every summer after that and usually discussed unpublished work of Tate (of which there was plenty!) frequently from typescripts by Lang. During the school year, the seminar continued and in 195960 it was dedicated to the theory of profinite groups, their cohomology groups, and Galois Cohomology. Here, though we didn't realize it, Tate was developing the étale cohomology of $\operatorname{Spec}(k)$-where $k$ is a field and this was very important as a test case for Grothendieck who was starting his drive toward the Weil Conjectures. 
In particular, we saw the theory of cohomological dimension and the application of these methods and techniques to local class field theory. The following summer we concentrated on duality, in particular for abelian varieties over local fields. There were many rough spots for us as neophytes because we had to absorb the idea of $\operatorname{Pic}(A)$ as an Ext, the Weil pairing, isogenies, and the connection of Cartier Duality (which we didn't really know) with all this stuff. I cannot communicate the excitement we felt, especially as it was mixed with the mystery engendered by our poor understanding.

During the academic year, Tate was also active with the circle (almost as a whole) and with its individual members. In particular, when several of us were taking Zariski's course on algebraic curves (Zariski always called this course: "Algebraic Functions of One Variable") and listening in on his seminar on abelian varieties, we needed Tate to explain to us the geometry (even the topology) behind all the algebra we saw from Zariski. This he did in his usual direct fashion and we were privileged to learn from two masters at once.

In the year '60-'61, the seminar was again very exciting. For, we studied elliptic curves and their arithmetic; in particular, Tate developed-before our eyes-the theory of what is now known as the "Tate Curve." There was the mysterious business of good reduction and various types of bad reduction. The structure of the formal group began to emerge as we studied the work of E. Lutz (in our secret seminar we looked at Mattuck's generalization) and we started our introduction to arithmetic algebraic geometry.

Many thesis topics came out of these seminars. Tate had the policy that, where possible, one should pick one's own thesis topic. He would comment on suitability and his approval meant you were more or less on your own-he had a minimal help policy, as well. This was, of course, all to the good. It was also tough on the student and served to inculcate the independence needed to pursue one's own ideas.

In the last year that I can speak about from personal knowledge, namely '61-'62, Grothendieck again visited. His course/seminar that year was a fair proportion of what is now the complete EGA IV. He went very fast, wrote practically nothing 
on the board (we were in effect taking dictation-a favorite subject of French students!) and left us to figure it out on our own later. Many hours were spent with Tate deciphering this material. Tate also lectured on his own way of seeing the material in EGA III on formal functions and the basic finiteness theorems for proper morphisms. The preparatory notes for the seminar on duality, run by Robin Hartshorne later, also seem to be derived from this visit. In addition, there was the well-attended seminar offered by Mike Artin on "Grothendieck Topologies"; so, we had plenty to do besides our own work.

I am certain the circle around Tate maintained its excitement and hectic pace after I graduated-it's hard to see how those two attributes could increase. What is clear is that the experience of mathematics at high level, in vast quantities, among interested and good students marked all of us for life. No mathematician in his role as passer on of mathematical culture and knowledge could have given us more. This is the heritage we took from Tate.

\section{Stephen S. Shatz}

Department of Mathematics

University of Pennsylvania

209 S. $33^{\text {rd }}$ Street

Philadelphia, PA 19104-6395 U.S.A.

E-mail: sss@sas.upenn.edu 